\title{
Products of Greek letter elements dug up from the third Morava stabilizer algebra
}

\author{
RYO KATO \\ KATSUMI SHIMOMURA
}

\begin{abstract}
In [3], Oka and the second author considered the cohomology of the second Morava stabilizer algebra to study nontriviality of the products of beta elements of the stable homotopy groups of spheres. In this paper, we use the cohomology of the third Morava stabilizer algebra to find nontrivial products of Greek letters of the stable homotopy groups of spheres: $\alpha_{1} \gamma_{t}, \beta_{2} \gamma_{t},\left\langle\alpha_{1}, \alpha_{1}, \beta_{p / p}^{p}\right\rangle \gamma_{t} \beta_{1}$ and $\left\langle\beta_{1}, p, \gamma_{t}\right\rangle$ for $t$ with $p \nmid t\left(t^{2}-1\right)$ for a prime number $p>5$.
\end{abstract}

$55 \mathrm{Q} 45 ; 55 \mathrm{Q} 51$

\section{Introduction}

Greek letter elements are well known generators of the stable homotopy groups of spheres localized at a prime $p$. Studying products among these elements is an interesting subject, and studied by several authors. For example, at an odd prime $p$, all products of alpha elements are trivial. In [3], we used $H^{*} S(2)$ to study nontriviality of the product of beta elements. In this paper, we use $H^{*} S(3)$ to find relations of Greek letters. The multiplicative structure of $H^{*} S(3)$ was given by Yamaguchi [7], but unfortunately, it has some typos. So here, our computation is based on Ravenel's [4].

Let $\beta_{p / p}$ be the generator of the $E_{2}$-term $E_{2}^{2, p^{2} q}(S)$ of the Adams-Novikov spectral sequence converging to the homotopy groups $\pi_{*}(S)$ of the sphere spectrum $S$. Hereafter, $q=2 p-2$ as usual. A relation given by Toda (see [4]) implies that $\beta_{p / p}$ dies in the Adams-Novikov spectral sequence at a prime $p>2$. At the prime two, $\beta_{2 / 2}^{2}=0$ by Miller, Ravenel and Wilson [2, Proposition 8.22], while at the primes three and five, Ravenel showed that $\beta_{p / p}^{p}$ survives to a homotopy element of $\pi_{*}(S)$ and $\alpha_{1} \beta_{p / p}^{p}=0$ for the generator $\alpha_{1}$ of $\pi_{q-1}(S)$. Here, we show the following:

1.1 Theorem At a prime $p>3, \beta_{p / p}^{p}$ survives to $\pi_{\left(p^{3}-1\right) q-2}(S)$ and $\alpha_{1} \beta_{p / p}^{p}=0$.

1.2 Corollary At a prime $p>3$, the Toda bracket $\left\langle\alpha_{1}, \alpha_{1}, \beta_{p / p}^{p}\right\rangle\left(=\alpha_{1} \beta_{p^{2} / p^{2}}\right)$ is defined. 
1.3 Remark It is already known that $\alpha_{1} \beta_{p^{2} / p^{2}}$ survives in the Adams-Novikov spectral sequence by the work of R Cohen [1]. Corollary 1.2 states that Cohen's element is a Toda bracket $\left\langle\alpha_{1}, \alpha_{1}, \beta_{p / p}^{p}\right\rangle$.

At the prime 3, Ravenel showed these results in [4].

Let $\beta_{1}, \beta_{2}$ and $\gamma_{t}(t>0)$ be the generators of Coker $J$ of dimensions $p q-2$, $(2 p+1) q-2$ and $\left(t p^{2}+(t-1) p+t-2\right) q-3$, respectively.

1.4 Theorem Let $p>5$, and $t$ be a positive integer with $p \nmid t\left(t^{2}-1\right)$. Then, the elements $\alpha_{1} \gamma_{t}, \beta_{2} \gamma_{t},\left\langle\alpha_{1}, \alpha_{1}, \beta_{p / p}^{p}\right\rangle \beta_{1} \gamma_{t}$ and $\left\langle\beta_{1}, p, \gamma_{t}\right\rangle$ generate subgroups of the stable homotopy groups of spheres isomorphic to $\mathbb{Z} / p$. Besides, even in the case $p \mid(t+1)$, the elements $\beta_{1} \gamma_{t}$ and $\left\langle\beta_{1}, p, \gamma_{t}\right\rangle$ are generators of order $p$.

Note that $\left\langle\beta_{1}, p, \gamma_{t}\right\rangle=\left\langle\gamma_{t}, p, \beta_{1}\right\rangle$. Also, if $t=1$, then $\left\langle\gamma_{1}, p, \beta_{1}\right\rangle=0$, while $\beta_{2} \gamma_{1}$ is nontrivial (see Section 5).

From here on, we assume that the prime number $p$ is greater than three.

\section{$2 \quad H^{*} S(3)$ revisited}

We begin with recalling some notation from Ravenel's green book [4]. Let $B P$ denote the Brown-Peterson spectrum. Then, the pair

$$
\left(B P_{*}, B P_{*}(B P)\right)=\left(\mathbb{Z}_{(p)}\left[v_{1}, v_{2}, \ldots\right], B P_{*}\left[t_{1}, t_{2}, \ldots\right]\right)
$$

is a Hopf algebroid. Here, the degrees of $v_{i}$ and $t_{i}$ are $2 p^{i}-2$. The structure maps act as follows:

$$
\begin{aligned}
\eta_{R}\left(v_{1}\right) & =v_{1}+p t_{1} \\
\eta_{R}\left(v_{2}\right) & \equiv v_{2}+v_{1} t_{1}^{p}+p t_{2} \quad \bmod \left(p^{2}, v_{1}^{p}\right) \\
\eta_{R}\left(v_{3}\right) & \equiv v_{3}+v_{2} t_{1}^{p^{2}}+v_{1} t_{2}^{p}+p t_{3} \\
\Delta\left(t_{1}\right) & =t_{1} \otimes 1+1 \otimes t_{1} \quad-p v_{1} v_{2}^{p-1}\left(t_{2}+t_{1}^{p+1}\right) \quad \bmod \left(p^{2}, v_{1}^{2}, v_{2}^{p}\right) \\
\Delta\left(t_{2}\right) & =t_{2} \otimes 1+t_{1} \otimes t_{1}^{p}+1 \otimes t_{2}-v_{1} b_{10} \\
\Delta\left(t_{3}\right) & \equiv t_{3} \otimes 1+t_{2} \otimes t_{1}^{p^{2}}+t_{1} \otimes t_{2}^{p}+1 \otimes t_{3} \quad \bmod \left(v_{1}, v_{2}\right) \\
\Delta\left(t_{4}\right) & \equiv t_{4} \otimes 1+t_{3} \otimes t_{1}^{p^{3}}+t_{2} \otimes t_{2}^{p^{2}}+t_{1} \otimes t_{3}^{p}+1 \otimes t_{4} \\
\Delta\left(t_{5}\right) & =t_{5} \otimes 1+t_{4} \otimes t_{1}^{p^{4}}+t_{3} \otimes t_{2}^{p^{3}}+t_{2} \otimes t_{3}^{p^{2}}+t_{1} \otimes t_{4}^{p}+1 \otimes t_{5} \\
& -v_{3} b_{22}-v_{4} b_{13} \bmod \left(p, v_{1}, v_{2}\right)
\end{aligned}
$$


for

$$
\begin{aligned}
b_{1 k} & =\frac{1}{p}\left(\Delta\left(t_{1}\right)^{p^{k+1}}-t_{1}^{p^{k+1}} \otimes 1-1 \otimes t_{1}^{p^{k+1}}\right) \\
& =\frac{1}{p} \sum_{i=1}^{p^{k+1}-1}\left(\begin{array}{c}
p^{k+1} \\
i
\end{array}\right) t_{1}^{i} \otimes t_{1}^{p^{k+1}-i} \\
b_{2 k} & =\frac{1}{p}\left(\Delta\left(t_{2}\right)^{\left.p^{k+1}-t_{2}^{p^{k+1}} \otimes 1-t_{1}^{p^{k+1}} \otimes t_{1}^{p^{k+2}}-1 \otimes t_{2}^{p^{k+1}}-v_{1}^{p^{k+1}} b_{1 k+1}\right)}\right.
\end{aligned}
$$

Let $K(3)_{*}=F_{p}\left[v_{3}, v_{3}^{-1}\right]$ have the $B P_{*}$-module structure given by $v_{i} v_{3}^{s}=v_{3}^{s} v_{i}=v_{3}^{s+1}$ if $i=3$, and $=0$ otherwise, and

$$
\begin{aligned}
\Sigma(3) & =K(3)_{*} \otimes_{B P_{*}} B P_{*}(B P) \otimes_{B P_{*}} K(3)_{*} \\
& =K(3)_{*}\left[t_{1}, t_{2}, \ldots\right] /\left(v_{3} t_{i}^{p^{3}}-v_{3}^{p^{i}} t_{i}: i>0\right) \quad(\text { by }[4,6.1 .16])
\end{aligned}
$$

is the Hopf algebra with structure inherited from $B P_{*}(B P)$. Define the Hopf algebra $S(3)$ by $S(3)=\Sigma(3) \otimes_{K(3)_{*}} F_{p}$, where $K(3)_{*}$ acts on $F_{p}$ by $v_{3} \cdot 1=1$. Then,

$$
S(3)=F_{p}\left[t_{1}, t_{2}, \ldots\right] /\left(t_{i}^{p^{3}}-t_{i}: i>0\right) .
$$

Now we abbreviate $\operatorname{Ext}_{S(3)}\left(F_{p}, F_{p}\right)$ to $H^{*} S(3)$.

Consider integers $d_{i}\left(=d_{3, i}\right.$ in $\left.[4,6.3 .1]\right)$

$$
d_{i}= \begin{cases}0 & i \leq 0, \\ \max \left(i, p d_{i-3}\right) & i>0 .\end{cases}
$$

Then, there is a unique increasing filtration of the Hopf algebroid $S(3)$ with $\operatorname{deg} t_{i}^{p^{j}}=d_{i}$ for $0 \leq j<3$.

2.3 Theorem (Ravenel $[4,6.3 .2]$ ) The associated Hopf algebra $E^{0} S(3)$ is isomorphic to the truncated polynomial algebra of height $p$ on the elements $t_{i}^{p^{j}}$ for $i>0$ and $j \in \mathbb{Z} / 3$, with coproduct defined by

$$
\Delta\left(t_{i}^{p^{j}}\right)= \begin{cases}\sum_{k=0}^{i} t_{k}^{p^{j}} \otimes t_{i-k}^{p^{k+j}} & i \leq 3 \\ t_{i}^{p^{j}} \otimes 1+1 \otimes t_{i}^{p^{j}}+b_{i-3, j+2} & i>3 .\end{cases}
$$

Let $L(3)$ be the Lie algebra without restriction with basis $x_{i, j}$ for $i>0$ and $j \in \mathbb{Z} / 3$ and bracket given by

$$
\left[x_{i, j}, x_{k, l}\right]= \begin{cases}\delta_{i+j}^{l} x_{i+k, j}-\delta_{k+l}^{j} x_{i+k, l} & \text { for } i+k \leq 3 \\ 0 & \text { otherwise }\end{cases}
$$


where $\delta_{j}^{i}=1$ if $i \equiv j \bmod 3$ and 0 otherwise, and $L(3, k)$ the quotient of $L(3)$ obtained by setting $x_{i, j}=0$ for $i>k$. Then, Ravenel noticed in $[4,6.3 .8]$ :

2.4 Theorem $H^{*}(L(3, k))$ for $k \leq 3$ is the cohomology of the exterior complex $E\left(h_{i, j}\right)$ on one-dimensional generators $h_{i, j}$ with $i \leq k$ and $j \in \mathbb{Z} / 3$, with coboundary

$$
d\left(h_{i, j}\right)=\sum_{s=1}^{i-1} h_{s, j} h_{i-s, s+j} .
$$

From now on, we abbreviate $h_{i, j}$ to $h_{i j}$, and $h_{1 j}$ to $h_{j}$.

Under the above filtration, Ravenel constructed the May spectral sequences:

2.5 Theorem (Ravenel $[4,6.3 .4,6.3 .5])$ There are spectral sequences

(a) $E_{2}=H^{*}(L(3,3)) \Longrightarrow H^{*}\left(E_{0} S(3)\right)$,

(b) $E_{2}=H^{*}\left(E_{0} S(3)\right) \Longrightarrow H^{*}(S(3))$.

Since these spectral sequences collapse, $H^{*} S(3)$ is additively isomorphic to $H^{*} L(3,3)$. Therefore, we have a projection

$$
\pi: H^{*} S(3) \rightarrow E^{0} H^{*} S(3)=H^{*}\left(E_{0} S(3)\right)=H^{*} L(3,3) .
$$

Note that the Massey product $\left\langle h_{i}, h_{i+1}, h_{i+2}, h_{i}\right\rangle$ is homologous to $v_{3}^{(2-p) p^{i}} b_{i+2}$ represented by $v_{3}^{(2-p) p^{i}} b_{1, i+2}$ of (2.2), and $\pi$ assigns the Massey product to $b_{i+2} \in$ $H^{*} L(3,3)$. Ravenel determined in [4, 6.3.34] the additive structure of $H^{*} L(3,3)$. In particular, we have the following:

2.7 Theorem $H^{*} L(3,3)$ contains submodules generated by the linear independent elements

$$
h_{1} k_{1} \zeta_{3}, \quad b_{0} k_{1} \zeta_{3}, \quad h_{0} l, \quad k_{0} l, \quad h_{0} b_{0} b_{2} l \quad \text { and } h_{1} l \text {. }
$$

Here, $l=h_{2} h_{21} h_{30}, k_{i}=h_{2 i} h_{i+1}(i=0,1), b_{0}=h_{1} h_{32}+h_{21} h_{20}+h_{31} h_{1}$, $b_{2}=h_{0} h_{31}+h_{20} h_{22}+h_{30} h_{0}$ and $\zeta_{3}=h_{30}+h_{31}+h_{32}$.

Proof In the table of the proof of [4, 6.3.34], we find the elements

$$
h_{0}, \quad h_{1}, \quad k_{0}, \quad b_{0}, \quad b_{2}, \quad l \quad l^{\prime}=h_{0} h_{22} h_{31}, \quad \text { and } \zeta_{3} \text {, }
$$

along with the first element $h_{1} k_{1} \zeta_{3}$ of the theorem. We also have the element $-h_{1} k_{1} h_{30}=h_{1} h_{2} h_{21} h_{30}$ in the table, which is the last element $h_{1} l$ of the theorem. Also in the table are $h_{1} k_{1} h_{31}$ and $h_{1} k_{1} h_{32}$. We see that $b_{0} k_{1}=-h_{1} k_{1} h_{31}+h_{1} k_{1} h_{32}$ and so the second element is given by $b_{0} k_{1} \zeta_{3}=-h_{1} k_{1} h_{31} \zeta_{3}+h_{1} k_{1} h_{32} \zeta_{3}$. 
The element $h_{0} b_{0} b_{2} l \zeta_{3}$ is computed as

$$
\begin{array}{r}
h_{0} h_{2} h_{21} h_{30}\left(h_{1} h_{32}+h_{21} h_{20}+h_{31} h_{1}\right)\left(h_{0} h_{31}+h_{20} h_{22}+h_{30} h_{0}\right)\left(h_{30}+h_{31}+h_{32}\right) \\
-2 h_{0} h_{1} h_{2} h_{20} h_{21} h_{22} h_{30} h_{31} h_{32} .
\end{array}
$$

Therefore, $h_{0} b_{0} b_{2} l$ is the dual of the generator $-\frac{1}{2} \zeta_{3}$, and the elements $h_{0} b_{0} b_{2} l$ and $h_{0} l$ are generators. Similarly, a computation

$$
\begin{aligned}
k_{0} l l^{\prime} \zeta_{3} & =h_{20} h_{1} h_{2} h_{21} h_{30} h_{0} h_{22} h_{31}\left(h_{30}+h_{31}+h_{32}\right) \\
& =h_{0} h_{1} h_{2} h_{20} h_{21} h_{22} h_{30} h_{31} h_{32}
\end{aligned}
$$

shows that $k_{0} l$ is the dual of the generator $l^{\prime} \zeta_{3}$.

2.8 Lemma In $H^{*} L(3,3), h_{0} k_{1}=0$ and $k_{0} k_{1}=0$.

Proof From the proof of [4, 6.3.34], we read off the relations $h_{0} k_{1}=e_{30} h_{2}$ and $k_{0} k_{1}=e_{30} g_{1}$ in $H^{*} L(3,2)$. Since $e_{30}$ cobounds $h_{30}$ in $H^{*} L(3,3)$, the lemma follows.

\section{Greek letter elements}

Let $E_{r}^{s, t}(X)$ denote the $E_{r}$-term of the Adams-Novikov spectral sequence converging to the homotopy group $\pi_{t-s}(X)$ of a spectrum $X$. Then the $E_{2}$-term is $\operatorname{Ext}_{B P_{*}(B P)}\left(B P_{*}, B P_{*}(X)\right)$. We here consider the Ext-group $\operatorname{Ext}_{B P_{*}(B P)}\left(B P_{*}, M\right)$ for a $B P_{*}(B P)$-comodule $M$ as the cohomology of the cobar complex $\Omega_{B P_{*}(B P)}^{*} M$ (cf [2]). Consider a sequence $A=\left(a_{0}, a_{1}, \ldots, a_{n}\right)$ of nonnegative integers so that the sequence $p^{a_{0}}, v_{1}^{a_{1}}, \ldots, v_{n}^{a_{n}}$ is invariant and regular. For such a sequence $A$, Miller, Ravenel and Wilson introduced in [2] $n$-th Greek letter elements $\eta_{S(A)}^{(n)}$ in the Adams-Novikov $E_{2}$-term $E_{2}^{n, t(A)}(S)$ by

$$
\eta_{s(A)}^{(n)}=\delta_{A, 1} \cdots \delta_{A, n}\left(v_{n}^{a_{n}}\right) \in E_{2}^{n, t(A)}(S)
$$

for

$$
v_{n}^{a_{n}} \in \operatorname{Ext}_{B P_{*}(B P)}^{0,2 a_{n}\left(p^{n}-1\right)}\left(B P_{*}, B P_{*} / I(A, n)\right) .
$$

Here, $s(A)=a_{n} / a_{n-1}, a_{n-2}, \ldots, a_{0}$ and $t(A)=2 a_{n}\left(p^{n}-1\right)-2 \sum_{i=0}^{n-1} a_{i}\left(p^{i}-1\right)$, $I(A, k)$ denotes the ideal of $B P_{*}$ generated by $p^{a_{0}}, v_{1}^{a_{1}}, \ldots, v_{k-1}^{a_{k-1}}$, and $\delta_{A, k+1}$ is the connecting homomorphism associated to the short exact sequence

$$
0 \rightarrow B P_{*} / I(A, k) \stackrel{v_{k}^{a_{k}}}{\longrightarrow} B P_{*} / I(A, k) \rightarrow B P_{*} / I(A, k+1) \rightarrow 0 .
$$


In particular, we write $\alpha=\eta^{(1)}, \beta=\eta^{(2)}$ and $\gamma=\eta^{(3)}$. So far, only when $n \leq 3$, many conditions for that Greek letter elements survives to homotopy elements are known. We abbreviate $\eta_{s(A)}^{(n)}$ to $\eta_{a_{n}}^{(n)}$ if $A=\left(1, \ldots, 1, a_{n}\right)$ as usual. For example, we consider $\beta$-elements defined by

$$
\begin{gathered}
\beta_{s}=\delta_{(1,1), 1}\left(\beta_{s}^{\prime}\right) \in E_{2}^{2, t(1,1, s)}(S) \\
\text { for } \beta_{s}^{\prime}=\delta_{(1,1), 2}\left(v_{2}^{s}\right) \in E_{2}^{1, t(1,1, s)}(V(0)), \\
\beta_{p^{i} / p^{i}}=\beta_{p^{i} / p^{i}, 1}=\delta_{\left(1, p^{i}\right), 1} \delta_{\left(1, p^{i}\right), 2}\left(v_{2}^{p^{i}}\right) \in E_{2}^{2, t\left(1, p^{i}, p^{i}\right)}(S) .
\end{gathered}
$$

Hereafter we assume that the prime $p$ is greater than three. We have the Smith-Toda spectrum $V(k)$ for $k=0,1,2$ defined by the cofiber sequences

$$
\begin{gathered}
S \stackrel{p}{\rightarrow} S \stackrel{i}{\rightarrow} V(0) \stackrel{j}{\rightarrow} \Sigma S, \\
\Sigma^{q} V(0) \stackrel{\alpha}{\rightarrow} V(0) \stackrel{i_{1}}{\rightarrow} V(1) \stackrel{j_{1}}{\rightarrow} \Sigma^{q+1} V(0), \\
\Sigma^{(p+1) q} V(1) \stackrel{\beta}{\rightarrow} V(1) \stackrel{i_{2}}{\rightarrow} V(2) \stackrel{j_{2}}{\rightarrow} \Sigma^{(p+1) q+1} V(1) .
\end{gathered}
$$

Here, $\alpha \in[V(0), V(0)]_{q}$ is the Adams map and $\beta \in[V(1), V(1)]_{(p+1) q}$ is the $v_{2}-$ periodic element due to L Smith. Note that the $B P_{*}$-homology of these spectra are $B P_{*}(V(k))=B P_{*} / I_{k+1}$ for the ideal $I_{k}$ of $B P_{*}$ generated by $v_{i}$ for $0 \leq i<k$ with $v_{0}=p$. We consider the Bousfield-Ravenel localization functor $L_{3}$ with respect to $v_{3}^{-1} B P$. The $E_{2}$-term $E_{2}^{*}\left(L_{3} V(2)\right)$ of $L_{3} V(2)$ is isomorphic to $K(3)_{*} \otimes H^{*} S(3)$, whose structure is given in [4] (see also [7]), and we consider the composite

$$
r: E_{2}^{*}(S) \stackrel{\iota_{*}}{\rightarrow} E_{2}^{*}(V(2)) \stackrel{\eta}{\rightarrow} E_{2}^{*}\left(L_{3} V(2)\right) \stackrel{\rho}{\rightarrow} H^{*}(S(3)) \stackrel{\pi}{\rightarrow} H^{*} L(3,3) .
$$

Here the first map is induced from the inclusion $\iota: S \rightarrow V(2)$ to the bottom cell, the second is from the localization map, the third is obtained by setting $v_{3}=1$ and the last is the projection (2.6).

3.4 Lemma The map $r$ assigns the Greek letter elements as follows:

$$
\begin{aligned}
& r\left(\alpha_{1}\right)=h_{0}, \quad r\left(\beta_{1}\right)=-b_{0}, \quad r\left(\beta_{2}\right)=2 k_{0}, \\
& r\left(\gamma_{t}\right)=-t\left(t^{2}-1\right) l-t(t-1) k_{1} \zeta_{3} \quad \text { and } \quad r\left(\beta_{p / p}\right)=-b_{1} .
\end{aligned}
$$

We also have $\beta_{1}^{\prime}=h_{1}-v_{1}^{p-1} h_{0} \in E_{2}^{1, p q}(V(0))$ for the generators $h_{i}$ of $E_{2}^{1, p^{i} q}(V(0))$ represented by $t_{1}^{p^{i}}$. 
Proof First we consider the images of the Greek letter elements under the map $\iota_{*}: E_{2}^{*}(S) \rightarrow E_{2}^{*}(V(2))$. In the cobar complex $\Omega_{B P_{*}(B P)}^{*} B P_{*}$, by $(2.1)$,

$$
\begin{aligned}
& d\left(v_{1}\right)=p t_{1}, \quad d\left(v_{2}^{p^{i}}\right) \equiv v_{1}^{p^{i}} t_{1}^{p^{i+1}}-v_{1}^{p^{i+1}} t_{1}^{p^{i}} \quad \bmod (p) \text { for } i \geq 0, \\
& d\left(v_{2}^{2}\right) \equiv 2 v_{1} v_{2} t_{1}^{p}+v_{1}^{2} t_{1}^{2 p} \quad \bmod \left(p, v_{1}^{p}\right), \\
& d\left(v_{3}^{t}\right) \equiv t v_{2} v_{3}^{t-1} t_{1}^{p^{2}}+\left(\begin{array}{l}
t \\
2
\end{array}\right) v_{2}^{2} v_{3}^{t-2} t_{1}^{2 p^{2}}+\left(\begin{array}{l}
t \\
3
\end{array}\right) v_{2}^{3} v_{3}^{t-3} t_{1}^{3 p^{2}} \quad \bmod \left(p, v_{1}, v_{2}^{4}\right),
\end{aligned}
$$

which imply

$$
\begin{array}{rlrl}
\delta_{(1), 1}\left(v_{1}\right) & =\left[t_{1}\right], & \delta_{(1,1), 2}\left(v_{2}\right)=\left[t_{1}^{p}-v_{1}^{p-1} t_{1}\right], \\
\delta_{(1,1), 2}\left(v_{2}^{2}\right) & =\left[2 v_{2} t_{1}^{p}+v_{1} t_{1}^{2 p}+v_{1}^{p-1} y\right], & \delta_{(1, p), 2}\left(v_{2}^{p}\right)=\left[t_{1}^{p^{2}}-v_{1}^{p^{2}-p} t_{1}^{p}\right], \\
\delta_{(1,1,1), 3}\left(v_{3}^{t}\right) & =\left[t v_{3}^{t-1} t_{1}^{p^{2}}+\left(\begin{array}{l}
t \\
2
\end{array}\right) v_{2} v_{3}^{t-2} t_{1}^{2 p^{2}}+\left(\begin{array}{l}
t \\
3
\end{array}\right) v_{2}^{2} v_{3}^{t-3} t_{1}^{3 p^{2}}+v_{2}^{3} z\right]=\bar{\gamma}_{t},
\end{array}
$$

for cochains $y \in \Omega_{B P_{*}(B P)}^{1} B P_{*} /(p)$ and $z \in \Omega_{B P_{*}(B P)}^{1} B P_{*} /\left(p, v_{1}\right)$. Here, $[x]$ denotes a cohomology class represented by a cocycle $x$. The first one shows $\alpha_{1_{k}}=h_{0}$, and the second gives the last statement of the lemma. We further see that $d\left(t_{1}^{p^{k}}\right)=-p b_{1 k-1}$ for $k \geq 1$ and $d\left(v_{k}\right) \equiv p t_{k} \bmod I((2,1,1), k)$ for $k=2,3$ by $(2.1)$ in $\in \Omega_{B P_{*}(B P)}^{1} B P_{*}$. Moreover, $\left[b_{1 k}\right]$ 's are assigned to $b_{k}$ in $H^{*} L(3,3)$ under the projection $\pi$, and we obtain

$$
\begin{aligned}
& r \delta_{\left(1, p^{k-1}\right), 1}\left(h_{k}-v_{1}^{p^{k}-p^{k-1}} h_{k-1}\right)=-b_{k-1} \quad \text { for } k=1,2, \\
& r \delta_{(1,1), 1}\left(\left[2 v_{2} t_{1}^{p}+v_{1} t_{1}^{2 p}\right]\right)=2 k_{0}, \\
& \delta_{(1,1,1), 2}\left(\bar{\gamma}_{t}\right)=\left[t(t-1) v_{3}^{t-2} t_{2}^{p} \otimes t_{1}^{p^{2}}+\left(\begin{array}{c}
t \\
2
\end{array}\right) v_{3}^{t-2} t_{1}^{p} \otimes t_{1}^{2 p^{2}}+w\right]=\gamma_{t}^{\prime}, \\
& r \delta_{(1,1,1), 1}\left(\gamma_{t}^{\prime}\right)=t(t-1)(t-2) h_{30} k_{1}+t(t-1) r \delta_{(1,1,1), 1}\left(\left[t_{2}^{p} \otimes t_{1}^{p^{2}}+\frac{1}{2} t_{1}^{p} \otimes t_{1}^{2 p^{2}}\right]\right) .
\end{aligned}
$$

Here, $w$ is a linear combination of terms in the ideal $\left(v_{1}, v_{2}\right)^{2}$. Thus the relations other than $r\left(\gamma_{t}\right)$ follows.

We note that $b_{20}$ in (2.2) corresponds to $h_{21} h_{30}+h_{31} h_{21}$ by $\Delta\left(t_{5}\right)^{p}$ in (2.1). Since $d\left(t_{2}^{p}\right)=-t_{1}^{p} \otimes t_{1}^{p^{2}}+v_{1}^{p} b_{11}-p b_{20}$ by (2.1), we obtain $r \delta_{(1,1,1), 1}\left(\left[t_{2}^{p} \otimes t_{1}^{p^{2}}+\frac{1}{2} t_{1}^{p} \otimes t_{1}^{2 p^{2}}\right]\right)=-\left(h_{21} h_{30}+h_{31} h_{21}\right) h_{2}+h_{21} b_{1}=-3 l-k_{1} \zeta_{3}$, which shows the relation on $r\left(\gamma_{t}\right)$.

Recall the cofiber sequences (3.3) and the $v_{3}$-periodic element $\gamma \in[V(2), V(2)]_{q_{3}}$ $\left(q_{3}=\left(p^{2}+p+1\right) q\right)$ due to $\mathrm{H}$ Toda. Then, the Greek letter elements in homotopy are 
defined by

$$
\alpha_{t}=j \alpha^{t} i, \quad \beta_{t}=j \beta_{t}^{\prime} \quad \text { for } \beta_{t}^{\prime}=j_{1} \beta^{t} i_{1} i \quad \text { and } \quad \gamma_{t}=j j_{1} j_{2} \gamma^{t} i_{2} i_{1} i
$$

for $t>0$, and the Greek elements in the $E_{2}$-term survives to the same named one in homotopy by the Geometric Boundary Theorem (cf [4]).

Proof of Theorem 1.4 We begin with noticing that the element $b_{i}$ in $H^{*} L(3,3)$ is the image of the Massey product $\left\langle h_{i}, h_{i+1}, h_{i+2}, h_{i}\right\rangle$ under $\pi$, which is homologous to $b_{i}$ represented by $b_{1 i}$ in (2.2). We further note that the Toda brackets $\left\langle\alpha_{1}, \alpha_{1}, \beta_{p / p}^{p}\right\rangle$ and $\left\langle\beta_{1}, p, \gamma_{t}\right\rangle$ are detected by $\alpha_{1} b_{2}$ and $h_{1} \gamma_{t}$ of $E_{2}^{*}(S)$, respectively. Indeed, in the first bracket, $d_{2 p-1}\left(b_{2}\right)=\alpha_{1} \beta_{p / p}^{p}$ by Corollary 4.4 below, and in the second bracket, $\left\langle\beta_{1}, p, \gamma_{t}\right\rangle=j\left\langle\beta_{1}^{\prime}, p, \gamma_{t}\right\rangle$. Under the condition on $t$, Lemmas 3.4, 2.7 and 2.8 imply that each element of $\alpha_{1} \gamma_{t}, \beta_{2} \gamma_{t}, \alpha_{1} b_{2} \gamma_{t} \beta_{1}$ and $h_{1} \gamma_{t}$, as well as $\beta_{1} \gamma_{t}$, generates a submodule isomorphic to $\mathbb{Z} / p$ of the $E_{2}$-term $E_{2}^{*}(S)$. These are, of course, permanent cycles, and nothing kills them in the Adams-Novikov spectral sequence since each element has a filtration degree less than $2 p-1$.

\section{$4 \beta_{p / p}^{p}$ in the homotopy of spheres}

Let $X$ and $\bar{X}$ be the $(p-1) q-$ and $(p-2) q-$ skeletons of the Brown-Peterson spectrum $B P$. Then, we have the cofiber sequences

$$
S \stackrel{\iota}{\rightarrow} X \stackrel{\kappa}{\rightarrow} \Sigma^{q} \bar{X} \stackrel{\lambda}{\rightarrow} S^{1} \quad \text { and } \quad \bar{X} \stackrel{\iota^{\prime}}{\rightarrow} X \stackrel{\kappa^{\prime}}{\rightarrow} S^{(p-1) q} \stackrel{\lambda^{\prime}}{\rightarrow} \Sigma \bar{X} .
$$

Then,

$$
B P_{*}(X)=B P_{*}[x] /\left(x^{p}\right) \quad \text { and } \quad B P_{*}(\bar{X})=B P_{*}[x] /\left(x^{p-1}\right)
$$

as subcomodules of $B P_{*}(B P)$, where $x$ corresponds to $t_{1}$. From [4, Chapter 7], we read off the following:

(4.2) $b_{1}^{p}=0 \in E_{2}^{2 p, p^{3} q}(X)$, which implies

$$
E_{2}^{2 s+e, t q}(X)=0 \quad \text { if } s \geq p \text { and } t<(s-1) p^{2}+(s+1+e) p .
$$

4.3 Lemma $b_{0}: E_{2}^{2 s+e, t q}(S) \rightarrow E_{2}^{2 s+2+e,(t+p) q}(S)$ is monomorphic if $s \geq p$ and $t \leq(s-1) p^{2}+(s+e) p$.

Proof Note that $b_{0}=\lambda \lambda^{\prime}$, and the lemma follows from (4.2) and the exact sequences

$$
\begin{gathered}
E_{2}^{2 s+e,(t+p-1) q}(X) \stackrel{\kappa^{\prime}}{\rightarrow} E_{2}^{2 s+e, t q}(S) \stackrel{\lambda^{\prime}}{\rightarrow} E_{2}^{2 s+1+e,(t+p-1) q}(\bar{X}), \\
E_{2}^{2 s+e+1,(t+p) q}(X) \rightarrow E_{2}^{2 s+e+1,(t+p-1) q}(\bar{X}) \stackrel{\lambda}{\rightarrow} E_{2}^{2 s+2+e,(t+p) q}(S)
\end{gathered}
$$

induced from the cofiber sequences in (4.1). 
Ravenel showed that $d_{2 p-1}\left(\beta_{p^{2} / p^{2}}\right) \equiv \alpha_{1} \beta_{p / p}^{p} \bmod \operatorname{Ker} \beta_{1}^{p}$ in the Adams-Novikov spectral sequence for $\pi_{*}(S)[4,6.4 .1]$. Here, the mapping $\beta_{1}^{p}$ on $E_{2}^{2 p+1,\left(p^{3}+1\right) q}(S)$ is a monomorphism by Lemma 4.3:

4.4 Corollary In the Adams-Novikov spectral sequence for $\pi_{*}(S)$,

$$
d_{2 p-1}\left(\beta_{p^{2} / p^{2}}\right)=\alpha_{1} \beta_{p / p}^{p} \in E_{2 p-1}^{2 p+1,\left(p^{3}+1\right) q}(S)=E_{2}^{2 p+1,\left(p^{3}+1\right) q}(S) .
$$

Proof of Theorem 1.1 Consider the first cofiber sequence in (4.1). The AdamsNovikov $E_{2}$-term $E_{2}^{s q+3,\left(p^{3}+s\right) q}(X)$ vanishes for $s>0$ by (4.2), so the element $\iota_{*}\left(\beta_{p^{2} / p^{2}}\right) \in E_{2}^{2, p^{3} q}(X)$ survives to a homotopy element $X_{p^{2} / p^{2}} \in \pi_{*}(X)$. In general, we see that:

Let $\bar{l}: S \rightarrow \bar{X}$ denote the inclusion to the bottom cell. Then, $\lambda_{*} \bar{l}(x)=\alpha_{1} x$ for $x \in E_{2}^{*}(S)$.

Put $\bar{\beta}_{p / p}=\bar{\iota}_{*}\left(\beta_{p / p}\right) \in E_{2}^{2, p^{2} q}(\bar{X})$, and we see that $\lambda_{*}\left(\bar{\beta}_{p / p}^{p}\right)=\alpha_{1} \beta_{p / p}^{p}$, and so we see that $\bar{\beta}_{p / p}^{p}$ detects an essential homotopy element $\kappa_{*}\left({ }^{X} \beta_{p^{2} / p^{2}}\right) \in \pi_{*}(\bar{X})$ by Corollary 4.4 and Shimomura [5], which we also denote by $\bar{\beta}_{p / p}^{p}$.

Now turn to the second cofiber sequence in (4.1). The relation $b_{1}^{p}=0$ of (4.2) yields a cochain $y=\sum_{i=0}^{p-1} x^{i} y_{i} \in \Omega^{2 p-1} B P_{*}(X)$ such that $d(y)=b_{1}^{p}$, where $y_{i} \in \Omega^{2 p-1} B P_{*}$. It follows that $d(\bar{y})=b_{1}^{p}-d\left(x^{p-1}\right) y_{p-1} \in \Omega^{2 p} B P_{*}(\bar{X})$ for $\bar{y}=$ $\sum_{i=0}^{p-2} x^{i} y_{i} \in \Omega^{2 p-1} B P_{*}(\bar{X})$. In particular $d\left(y_{p-1}\right)=0 \in \Omega^{2 p-1} B P_{*}$ and $d\left(y_{p-2}\right)=$ $(1-p) t_{1} \otimes y_{p-1}$. By definition, these imply $\lambda_{*}^{\prime}\left(y_{p-1}\right)=b_{1}^{p}$. Consider the exact sequence obtained by applying the homotopy groups to the second cofiber sequence. Then, $\iota_{*}^{\prime}\left(\bar{\beta}_{p / p}^{p}\right)=0$ by (4.2), and so $\bar{\beta}_{p / p}^{p}$ must be pulled back to an element $\xi \in$ $\pi_{*}(S)$ detected by $y_{p-1}$. Since $b_{0}=\lambda \lambda^{\prime}, b_{0} y_{p-1}=h_{0} b_{1}^{p}$, and $\left\langle h_{0}, \ldots, h_{0}\right\rangle y_{p-1}=$ $h_{0}\left\langle h_{0}, \ldots, h_{0}, y_{p-1}\right\rangle$, we see that

$$
b_{1}^{p} \equiv\left\langle h_{0}, \ldots, h_{0}, y_{p-1}\right\rangle \not \equiv 0 \in E_{2}^{2 p, p^{3} q}(S) \bmod \operatorname{ker} h_{0} .
$$

Put $b_{1}^{p}=\left\langle h_{0}, \ldots, h_{0}, y_{p-1}\right\rangle+c$ for $c \in \operatorname{ker} h_{0} \subset E_{2}^{2 p, p^{3} q}(S)$. Then, $b_{1}^{p}-c$ survives to $\beta_{p / p}^{p} \in \pi_{*}(S)$.

The element $\alpha_{1} \beta_{p / p}^{p}$ is detected by $h_{0}\left(b_{1}^{p}-c\right)=h_{0} b_{1}^{p}$ in the Adams-Novikov $E_{2}-$ term, which is killed by $b_{2}$ by Corollary 4.4.

\section{Remarks}

\subsection{A relation on Toda bracket}

The relation $\left\langle\beta_{s}, p, \gamma_{t}\right\rangle=\left\langle\gamma_{t}, p, \beta_{s}\right\rangle$ follows immediately from results of Toda: By definition, $\left\langle\beta_{s}, p, \gamma_{t}\right\rangle=j \beta_{(s)} \gamma_{(t)} i$ and $\left\langle\gamma_{t}, p, \beta_{s}\right\rangle=j \gamma_{(t)} \beta_{(s)} i$ for $\beta_{(s)}=j_{1} \beta^{s} i_{1}$ 
and $\gamma_{(t)}=j_{1} j_{2} \gamma^{t} i_{2} i_{1}$. Since $V(2)$ and $V(3)$ are $V(0)$-module spectra, $\theta(\beta)=0$ and $\theta(\gamma)=0$ by Toda [6, Lemma 2.3]. Similarly, $\theta\left(i_{k}\right)=0$ and $\theta\left(j_{k}\right)=0$ for $k=1$, 2. Therefore, [6, Lemma 2.2] implies $\theta\left(\beta_{(s)}\right)=0$ and $\theta\left(\gamma_{(t)}\right)=0$. Therefore, $\beta_{(s)} \gamma_{(t)}=\gamma_{(t)} \beta_{(s)}$ by [6, Corollary 2.7] as desired.

\subsection{On the action of $\gamma_{1}$}

Note that $\gamma_{1}=\alpha_{1} \beta_{p-1}$. Then, $\alpha_{1} \gamma_{1}=\alpha_{1}^{2} \beta_{p-1}=0$ and $\left\langle\alpha_{1}, \alpha_{1}, \beta_{p / p}^{p}\right\rangle \beta_{1} \gamma_{1}=$ $-\alpha_{1}\left\langle\alpha_{1}, \alpha_{1}, \beta_{p / p}^{p}\right\rangle \beta_{1} \beta_{p-1}=-\left\langle\alpha_{1}, \alpha_{1}, \alpha_{1}\right\rangle \beta_{p / p}^{p} \beta_{1} \beta_{p-1}=0$ since $\left\langle\alpha_{1}, \alpha_{1}, \alpha_{1}\right\rangle=0$, and $\left\langle\gamma_{1}, p, \beta_{1}\right\rangle=\beta_{p-1}\left\langle\alpha_{1}, p, \beta_{1}\right\rangle=\beta_{p-1} j \underline{\alpha j_{1}} \beta i_{1} i=0$.

For $t \geq 2$,

$$
\begin{aligned}
\beta_{t} & =\delta_{(1,1), 1} \delta_{(1,1), 2}\left(v_{2}^{t}\right)=\delta_{(1,1), 1}\left(\left[t v_{2}^{t-1} t_{1}^{p}+\left(\begin{array}{l}
t \\
2
\end{array}\right) v_{1} v_{2}^{t-2} t_{1}^{2 p}+v_{1}^{2} x\right]\right) \\
& \equiv\left[t(t-1) v_{2}^{t-2} t_{2} \otimes t_{1}^{p}-t v_{2}^{t-1} b_{0}+\left(\begin{array}{c}
t \\
2
\end{array}\right) v_{2}^{t-2} t_{1} \otimes t_{1}^{2 p}\right] \bmod \left(p, v_{1}\right) \\
& \equiv t(t-1) v_{2}^{t-2} k_{0}-t v_{2}^{t-1} b_{0} \bmod \left(p, v_{1}\right)
\end{aligned}
$$

and $\alpha_{1} \beta_{2} \beta_{p-1} \in E_{2}^{5}\left(S^{0}\right)$ is projected to $h_{0}\left(2 k_{0}-2 v_{2} b_{0}\right)\left(2 v_{2}^{p-3} k_{0}+v_{2}^{p-2} b_{0}\right)=$ $-2 v_{2}^{p-2} h_{0} k_{0} b_{0}-2 h_{0} v_{2}^{p-1} b_{0}^{2}$ in $E_{2}^{5}(V(2))$ under the induced map $i_{*}$ from the inclusion $i: S^{0} \rightarrow V(2)$ to the bottom cell. Here, $k_{0}=\left[t_{2} \otimes t_{1}^{p}+\frac{1}{2} t_{1} \otimes t_{1}^{2 p}\right]$. Then, this element is detected by $-2 v_{2}^{p-2} k_{0} \in E_{1}^{3}=E_{2}^{2,\left(p^{2}+p-1\right) q}(X \wedge V(2))$ in the small descent spectral sequence. The killer of this element, if any, stays in the $E_{1}$-terms

$$
\begin{aligned}
E_{1}^{2}=E_{2}^{2,\left(p^{2}+p\right) q}(X \wedge V(2)), \quad E_{1}^{1}=E_{2}^{3,\left(p^{2}+2 p-1\right) q}(X \wedge V(2)), \\
E_{1}^{0}=E_{2}^{4,\left(p^{2}+2 p\right) q}(X \wedge V(2)) .
\end{aligned}
$$

These are zero, and we see that the product is not zero.

\section{References}

[1] R L Cohen, Odd primary infinite families in stable homotopy theory, Mem. Amer. Math. Soc. 30, no. 242, Amer. Math. Soc. (1981) MR603393

[2] H R Miller, D C Ravenel, W S Wilson, Periodic phenomena in the Adams-Novikov spectral sequence, Ann. of Math. 106 (1977) 469-516 MR0458423

[3] S Oka, K Shimomura, On products of the $\beta$-elements in the stable homotopy of spheres, Hiroshima Math. J. 12 (1982) 611-626 MR676562

[4] D C Ravenel, Complex cobordism and stable homotopy groups of spheres, Pure and Applied Math. 121, Academic Press, Orlando, FL (2000) MR860042 
[5] K Shimomura, On differential of a generalized Adams spectral sequence, J. Fac. Educ. Tottori Univ. (Nat. Sci.) 41 (1992) 119-131

[6] H Toda, Algebra of stable homotopy of $Z_{p}$-spaces and applications, J. Math. Kyoto Univ. 11 (1971) 197-251 MR0293631

[7] A Yamaguchi, The structure of the cohomology of Morava stabilizer algebra S(3), Osaka J. Math. 29 (1992) 347-359 MR1173994

Graduate school of Mathematics, Nagoya Unversity

Furo-cho, Chikusa-ku, Nagoya 464-8602, Japan

Department of Mathematics, Faculty of Science, Kochi University

2-5-1, Akebono, Kochi 780-8520, Japan

ryo_kato_1128@yahoo.co.jp, katsumi@kochi-u.ac.jp

Received: 1 August 2011 Revised: 29 November 2011 